\title{
Influence of Strong Acoustic Anisotropy on Reflection of Bulk Acoustic Waves in Crystals
}

\author{
N. Polikarpova* \\ Department of Physics, M.V. Lomonosov Moscow State University \\ 119992 Moscow, Russia
}

Propagation and reflection of plane elastic waves in the acousto-optic crystals tellurium dioxide and calomel as well as in the ferroelectric material barium titanate is examined in the paper. The reflection of the waves from a free and flat boundary separating the crystal and the vacuum is investigated in the (001) planes of $\mathrm{TeO}_{2}, \mathrm{Hg}_{2} \mathrm{Cl}_{2}$, and $\mathrm{BaTiO}_{3}$ in the case of glancing acoustic incidence on the boundary. The analysis shows that as much as two elastic waves may be reflected from the crystal surface. Energy flow of one of the reflected waves in paratellurite and calomel propagates in a quasi-back direction with respect to the incident energy flow, so that both energy flows are separated by an angle as narrow as a few degrees. It is found that a relative intensity of the unusually reflected wave in the acousto-optic crystals may be close to a unit in a wide variety of crystal cuts. Possible applications of the examined phenomena in acousto-optic devices are discussed in the presentation.

PACS numbers: 43.35.Sx, 78.20.Hp, 43.25.-x, 61.72.--y

\section{Introduction}

The paper describes physical phenomena that may be observed in a process of propagation and reflection of plane elastic waves in crystals. The new effects manifest themselves in the most impressive manner in the modern acousto-optic materials such as paratellurite $\mathrm{TeO}_{2}$ and calomel $\mathrm{Hg}_{2} \mathrm{Cl}_{2}$ as well as in other crystals possessing the extremely strong anisotropy of their elastic properties [1-9]. It should be noted that growth and fabrication of these unique crystalline materials was to a great extent stimulated by requirements of acousto-optics, optical communication, and laser technology [1-9]. In this paper, the acousto-optic material paratellurite $\mathrm{TeO}_{2}$ and calomel $\mathrm{Hg}_{2} \mathrm{Cl}_{2}$ were chosen for the analysis. These materials are characterized by the pronounced elastic anisotropy so that the value

*e-mail: polikarp@phys.msu.ru 
of the acoustic phase velocity $V$ strongly depends on a direction of propagation [3-9]. In particular, the slow-shear acoustic wave propagating in the $X Y$ plane at the angle $\theta=45^{\circ}$ relatively to the axis $X$ or $Y$ possesses the extraordinary low phase velocity values $V=616 \mathrm{~m} / \mathrm{s}$, for paratellurite, and $V=347 \mathrm{~m} / \mathrm{s}$, for calomel. On the other hand, the waves sent along the axes $X$ and $Y$ of the materials are characterized by the velocity value $V=3050 \mathrm{~m} / \mathrm{s}$, for paratellurite, and $V=1326 \mathrm{~m} / \mathrm{s}$, for calomel, that is $4-5$ times higher [1]. It should be noted that, in crystalline materials with a moderate elastic anisotropy, for example in $\mathrm{BaTiO}_{3}$, the ratio $r$ of the maximal and minimal phase velocity values does not exceed the factor of $r \leq 2.0-2.5[1]$.

The elastic waves propagating in the anisotropic crystals, such as paratellurite, and calomel are characterized by large walk-off angles $\psi$ between the phase $V$ and the group $V_{\mathrm{g}}$ velocities of ultrasound $[1-8,10-15]$. It may be shown that the walk-off angle $\psi$ between the acoustic wave vector $\boldsymbol{K}$ and the Poynting vector in these materials may exceed dozens of degrees, e.g. $\psi>70^{\circ}[1-8,15-18]$, while in $\mathrm{BaTiO}_{3}$, the angle is limited to $\psi>40^{\circ}$. This characteristic feature of the waves may cause the strange reflection of the acoustic energy incident on a free boundary separating the materials and the vacuum. In this paper, the unusual behavior of the waves was examined for the case of the acoustic propagation and reflection in the $X Y$ plane of $\mathrm{TeO}_{2}, \mathrm{Hg}_{2} \mathrm{Cl}_{2}$, and $\mathrm{BaTiO}_{3}$. The investigation, in its major part, was carried out in specimens of rectangular configuration. However, results of the analysis may be generalized over other media possessing the strong elastic anisotropy. Moreover, the revealed regular trends are typical of crystalline specimens of arbitrary configurations and not only of the rectangular configuration.

\section{Glancing incidence and reflection in tetragonal crystals}

The drawing in Fig. 1 shows the rectangular specimen of a crystal of the tetragonal structure and the piezoelectric transducer bonded to the side facet of the crystal. It is expected that the transducer generates a quasi-longitudinal or quasi-shear acoustic wave (1) in the crystal. It is assumed that the incident wave possesses the phase velocity $V_{1}$ and the corresponding wave vector $\boldsymbol{K}_{1}$ directed at the angle $\varphi$ relatively to the axis $X$ [100]. As illustrated, the acoustic beam (1) propagates in the material with the walk-off angle $\psi_{1}$ evaluated between the phase $V_{1}$ and group $V_{\mathrm{g} 1}$ velocities of ultrasound. After the propagation in the crystal, the acoustic energy is incident on the bottom facet of the specimen. This facet of the crystal serves as a border between the material and the vacuum. It should be emphasized that the acoustic energy is incident on the facet that is free, flat and homogeneous.

According to the prompts of everyday experience, it is reasonable to predict that the reflected beam (2) propagates from the bottom surface of the crystal away from the direction of the acoustic incidence. As seen in Fig. 1, the beam (2) is characterized by the obliquity angle $\psi_{2}$. This angle is evaluated between the 


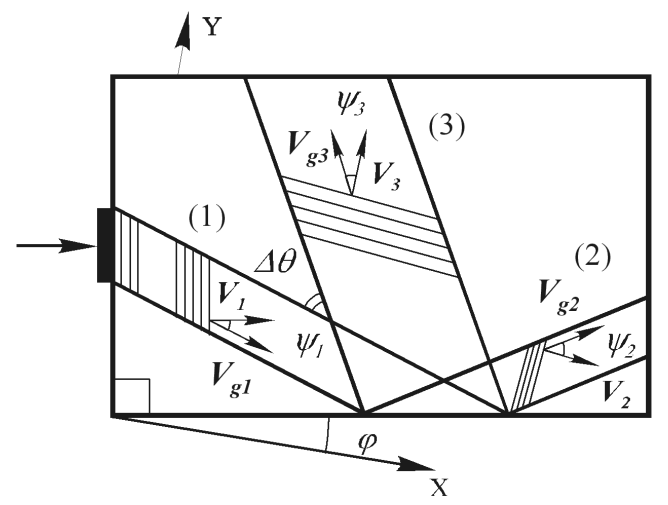

Fig. 1. General scheme of reflection in crystals with strong anisotropy.

wave vector $\boldsymbol{K}_{2}$ or the phase velocity vector $\boldsymbol{V}_{2}$, on the one hand, and the group velocity vector $\boldsymbol{V}_{\mathrm{g} 2}$, on the other hand. However, additionally to the traditionally reflected beam (2), there exists the unusually reflected beam (3). It possesses the phase velocity vector $\boldsymbol{V}_{3}$ corresponding to the wave vector $\boldsymbol{K}_{3}$. The extraordinary reflected beam (3) and the incident ray (1) are separated in space by the angle $\Delta \theta$. In the examined case of the acoustic reflection, one of the two reflected waves, in particular, the wave (3) may propagate approximately towards the incident acoustic ray, i.e. in back direction relatively to the direction of the incidence $[10-13,17-21]$.

\section{Spatial separation angle between two reflected waves}

In the $X Y$ plane of the crystals, the magnitudes of the acoustic walk-off angles of the three acoustic beams $\psi_{1}, \psi_{2}$ and $\psi_{3}$ were calculated for the directions of the incident wave propagation corresponding to the cut angle $0<\varphi<45^{\circ}$. During the analysis, the angles $\Delta \theta_{2}$ and $\Delta \theta_{3}$ separating in space the acoustic columns of the incident (1), the ordinary (2) and the extraordinary (3) reflected beams were determined for each value of the cut angle $\varphi$. Data in Fig. 2 demonstrate the calculated dependence of the separation angles $\Delta \theta_{2}$ and $\Delta \theta_{3}$ on the cut angle of the crystal for the paratellurite crystal. It may be seen in Fig. 2 that the angle $\Delta \theta_{3}$ occurs amazingly small $\Delta \theta_{3}<10^{\circ}$ over the wide range of the propagation angles $4^{\circ}<\varphi<32^{\circ}$. It means that there are no strict requirements on the orientation of the specimen if one intends to observe the peculiar reflection in the crystal. Data in Fig. 2 also represent the behavior of the spatial separation angle $\Delta \theta_{2}$ for the ordinary reflected wave (2). As proved by the analysis, this wave exists only at $0<\varphi \leq 24^{\circ}$. Similar calculations were made for the single crystal of calomel. The separation angle for the extraordinary reflected wave in this case, in a great variety of crystal cuts, does not also exceed the magnitude $\Delta \theta_{3}<10^{\circ}$.

The performed calculations demonstrate that the minimum value of the separation angle in tellurium dioxide occurs as low as $\Delta \theta_{3}=5.3^{\circ}$. It is quite likely 


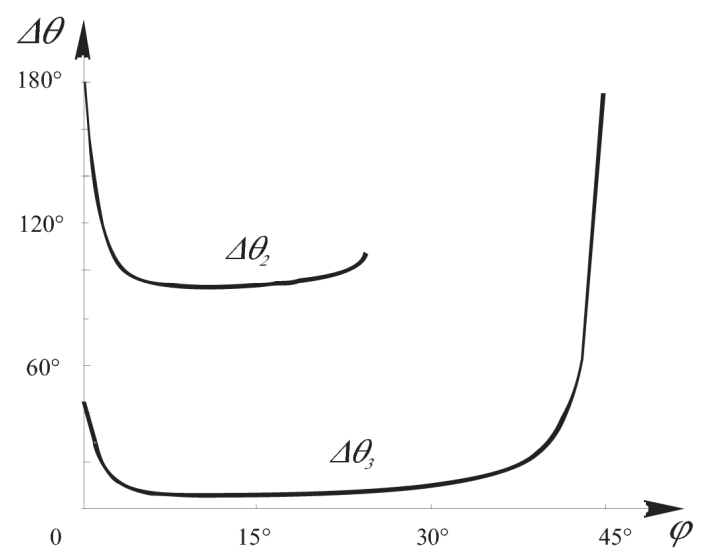

Fig. 2. Separation angle for the slow acoustic mode in single crystal $\mathrm{TeO}_{2}$.

that this unique case of the quasi-back reflection, that follows the glancing incidence, has not so far been examined in acoustics. The carried out research also proves that the revealed peculiarity is typical not only of tellurium dioxide but of all other materials possessing the strong anisotropy of their elastic properties. For example, the calculation proved that the minimum separation angle in the single crystal of calomel $\mathrm{Hg}_{2} \mathrm{Cl}_{2}$ is limited to $\Delta \theta_{3}=9.8^{\circ}$. For comparison, in the crystal of $\mathrm{BaTiO}_{3}$, the minimum value of the separation angle is as large as $\Delta \theta_{3}=73^{\circ}$. It means that the acoustic reflection in barium titanate takes place in forward, i.e. not back direction with respect to the incident energy flow.

The performed analysis was also extended over the cases of the glancing incidence and reflection of the fast, i.e. the quasi-longitudinal wave in the specimens. As found, one of the reflected waves is the quasi-shear acoustic mode, while another wave is the quasi-longitudinal mode. It was proved that the unusual reflection is typical of the incident quasi-longitudinal wave because the minimal separation angle in $\mathrm{TeO}_{2}$ is equal to $\Delta \theta_{3}=6.4^{\circ}$. In the crystal $\mathrm{Hg}_{2} \mathrm{Cl}_{2}$, this angle possesses the value $\Delta \theta_{3}=12.2^{\circ}$. On the other hand, in the materials without the strong anisotropy, such as $\mathrm{BaTiO}_{3}$, the separation angle is equal to $\Delta \theta_{3}=76^{\circ}$, i.e. up to dozens of degrees.

Therefore, on base of the carried out analysis, it is reasonable to conclude that the quasi-back reflection of the acoustic waves should be considered as a phenomenon typical of both incident acoustic modes in $\mathrm{TeO}_{2}$ and $\mathrm{Hg}_{2} \mathrm{Cl}_{2}$, i.e. the quasi-shear and quasi-longitudinal mode. Moreover, this phenomenon may be observed not only in the paratellurite and the calomel crystals but also in all elastic media possessing the strong anisotropy of the acoustic properties. On the other hand, the unusual effect is absent in media with a moderate and low elastic anisotropy. 


\section{Distribution of elastic energy over reflected waves}

In order to fulfill the analysis of the reflection, mutual distribution of the incident elastic energy over the two reflected waves in Fig. 1 was determined. The reflection coefficients $R_{2}$ and $R_{3}$ describing energy flows of the beams (2) and (3) were calculated for the purpose. The coefficients were defined as the ratio of the normal projections of the energy flows of the reflected and the incident beams $[1,10]$.

Data in Fig. 3 show the dependence of the reflection coefficients $R_{2}$ and $R_{3}$ on the cut angle $\varphi$ of the paratellurite crystal. It may be seen that there exist as much as four intervals of the angle $\varphi$ corresponding to a different behavior of the reflection coefficients in the specimen. These intervals are $0^{\circ}<\varphi \leq 3^{\circ}$, $3^{\circ}<\varphi \leq 23^{\circ}, 23^{\circ}<\varphi \leq 25^{\circ}$ and $25^{\circ}<\varphi \leq 45^{\circ}$. The analysis confirms that if the crystal is cut at the angle $\varphi$ slightly different from zero, then the major amount of the incident energy is reflected in form of the ordinary elastic wave, i.e. the wave (2) in Fig. 1. Growth of the angle $\varphi$ is accompanied by the rapid increase in the energy of the extraordinary reflected wave (3), while the intensity of the forward reflected wave (2) vanishes.

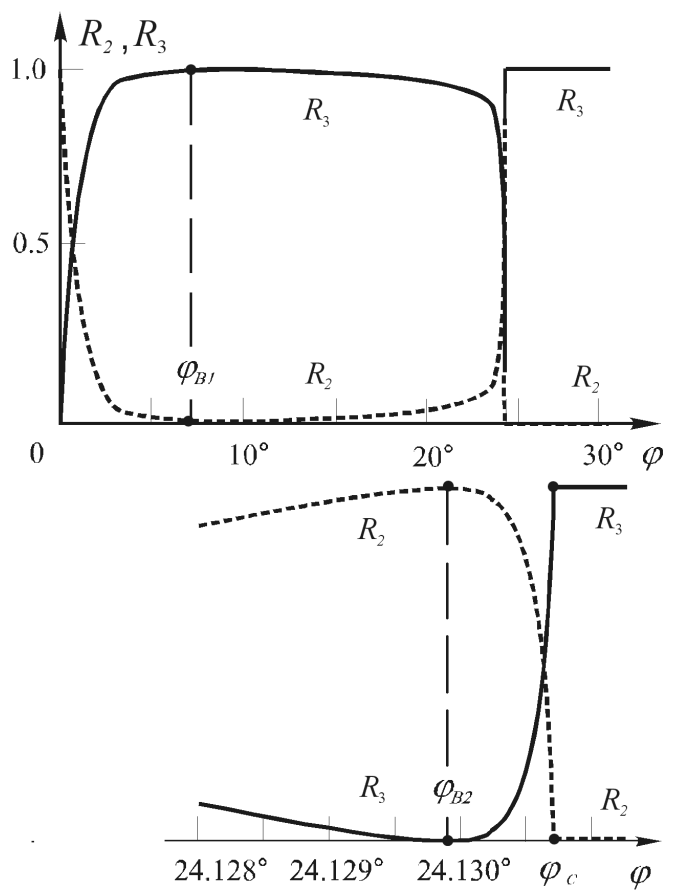

Fig. 3. Reflection coefficients of the slow acoustic mode in the $X Y$ plane of $\mathrm{TeO}_{2}$.

If the propagation angle $\varphi$ is limited in the range $3^{\circ}<\varphi \leq 23^{\circ}$ then the major amount of the elastic energy is concentrated in the extraordinary reflected 
ray. Calculation proves that, at the particular angle $\varphi_{\mathrm{B} 1}=7.5^{\circ}$, all the incident elastic power is reflected in the quasi-back direction because the coefficient $R_{3}=$ 1.0. Consequently, the angle $\varphi_{\mathrm{B} 1}$ may be defined as the Brewster angle, at which the reflected wave (2) is absent.

Analysis of data in Fig. 3 proves that the interval of angles $23^{\circ}<\varphi \leq 25^{\circ}$ is characterized by the extremely abrupt changes of the reflection coefficients. This interval includes two characteristic points, one of which is the Brewster angle $\varphi_{\mathrm{B} 2}$ while another one is the critical angle $\varphi_{\mathrm{c}}$. The carried out calculations indicate that, in tellurium dioxide, the magnitudes of the second Brewster and the critical angle are very close to each other $\varphi_{\mathrm{B} 2} \approx 24.130^{\circ}$ and $\varphi_{\mathrm{c}} \approx 24.131^{\circ}$. Consequently, some kind of unstable behavior of the waves may be predicted so that it is unlikely to distinguish between these two angles experimentally. As for the distribution of the elastic energy over the two reflected waves, it is predicted that, in the interval of the angles from $\varphi=23^{\circ}$ to $\varphi_{\mathrm{B} 2} \approx 24.13^{\circ}$, the reflection coefficient $R_{2}$ rapidly approaches a unity, while the energy flow of the extraordinary reflected beam vanishes. It means that a total reflection of the incident energy along the direction of the ordinary wave (2) may be expected in the specimen at the Brewster angle $\varphi=\varphi_{\mathrm{B} 2}$. On the contrary, a further increase in the cut angle from $\varphi=\varphi_{\mathrm{B} 2}$ to the critical angle $\varphi=\varphi_{\mathrm{c}}$ is accompanied by the abrupt drop of the energy of the ordinary wave so that $R_{2}=0$. It results in an inevitable jump of the energy in the back reflected wave (3) from $R_{3}=0$ to $R_{3}=1.0$.

As for the critical angle $\varphi_{\mathrm{c}}$, it may be concluded that all incident elastic energy is reflected from the boundary as the quasi-back wave (3). Moreover, the investigation confirms that the forward wave (2) disappears at $\varphi>\varphi_{\mathrm{c}}$. It may be expected that the ordinary reflected wave at $\alpha=\alpha_{\mathrm{c}}$ becomes inhomogeneous. It propagates with the energy flow directed along the border of the crystal. It is obvious that all cases of the reflection with $\varphi>\varphi_{\mathrm{c}}$ correspond to the single extraordinary reflected acoustic wave (3) possessing the reflection coefficient $R_{3}=1.0$. The energy flow of this wave is directed practically opposite relatively to the energy flow of the incident beam because the separation angle $\Delta \theta_{3}$ in Fig. 2 is limited to $\Delta \theta_{3} \leq 10^{\circ}$ over the wide range of the cut angles. In the crystal of calomel, the absolute magnitudes of the angles are slightly different from those in $\mathrm{TeO}_{2}$. However, in general, the behavior of the waves during the reflection remains unchanged. On the other hand, it was found that, in calomel, the first Brewster angle is absent because the maximal value of the reflection coefficient of the back reflected wave is equal to $R_{3}=0.93$. In other words, the anisotropy is not so strong as in paratellurite. Nevertheless, the anisotropy is still quite sounding so that the difference between the second Brewster angle and the critical angle $\varphi_{\mathrm{c}}=22.324^{\circ}$ in the calomel is negligible.

Such kind of the acoustic behavior in the materials possessing a moderate elastic anisotropy, for example in $\mathrm{BaTiO}_{3}$, may not be observed. This statement is confirmed by calculations of the reflection coefficients for the case of the quasi-shear 
waves propagating in barium titanate. It was found that the maximal magnitude of the reflection coefficient for the back wave in $\mathrm{BaTiO}_{3}$ is very low $R_{3}=0.05$. The analysis also confirms that the magnitudes of the Brewster and the critical angles in the ferroelectric material occur correspondingly equal to $\varphi_{\mathrm{B} 2}=8.12^{\circ}$ and $\varphi_{\mathrm{c}}=9.73^{\circ}$. Consequently, the difference between these two angles $1.6^{\circ}$, in comparison with the difference $0.01^{\circ}$ in the paratellurite or calomel, is quite noticeable. Finally, as mentioned, the absolute magnitudes of the reflection coefficients of the back reflected waves do not exceed $R_{3}<1 \%$. In this respect, the efficiency of the unusual reflection in $\mathrm{BaTiO}_{3}$ may not be compared to the efficiency $R_{3} \approx 1.0$ typical of the acousto-optic crystals. Moreover, the back reflected wave in barium titanate propagates far away from the energy flow of the incident energy flow. That is why the quasi-back reflection in the traditional materials practically does not exist.

\section{Conclusion}

It may be stated that propagation of acoustic waves in the crystalline materials possessing strong anisotropy of their elastic properties may be accompanied by a peculiar quasi-back reflection of acoustic energy from a free surface separating the crystals and the vacuum. The peculiarity of the reflection consists in the fact that the unusual reflection follows the glancing incidence of the elastic energy on a crystal facet. Relative intensity of the extraordinary reflected waves in such acousto-optic crystals as paratellurite and calomel may be as high as $100 \%$. These unique cases of the acoustic reflection are interesting not only from the point of view of fundamental knowledge but also of applied sciences. For example, new types of acousto-electronic and acousto-optic instruments may be designed on base of the examined effects. Tunable acousto-optic filters with collinear and non-collinear propagation of beams as well as acousto-electronic delay lines with low consumption of expensive crystalline materials are the most evident examples of the applications.

\section{References}

[1] B.A. Auld, Acoustic Fields and Waves in Solids, Robert Krieger Publ. Co., New York 1990.

[2] A. Goutzoulis, D. Pape, Design and Fabrication of Acousto-Optic Devices, Marcel Dekker Inc., New York 1994.

[3] I.C. Chang, Appl. Phys. Lett. 25, 323 (1974).

[4] J.C. Kastelik, M.G. Gazalet, C. Bruneel, E. Bridoux, J. Appl. Phys. 74, 2813 (1993).

[5] C.D. Tran, G.-C. Huang, Opt. Eng. 38, 1143 (1999).

[6] E.G. Lean, W.H. Chen, Appl. Phys. Lett. 35, 101 (1979).

[7] S.N. Antonov, A.V. Gerus, V.V. Proklov, Sov. Phys. Tech. 28, 984 (1983).

[8] V.B. Voloshinov, Ultrasonics 31, 333 (1993). 
[9] M. Gottlieb, A. Goutzoulis, N. Singh, Opt. Eng. 31, 2110 (1992).

[10] M.J.P. Musgrave, Geophys. J. Roy. Astron. Soc. 3, 406 (1960).

[11] E.G. Henneke II, J. Acoust. Soc. Am. 53, 1176 (1973).

[12] M.M. Shevelko, L.A. Yakovlev, Sov. Phys. Acoust. 20, 553 (1975).

[13] P.C. Waterman, Phys. Rev. 113, 1240 (1959).

[14] V.I. Pustovoit, I.S. Ravvin, L.A. Chernozatonskii, JETP Lett. 25, 144 (1977).

[15] V.B. Voloshinov, Opt. Eng. 31, 2089 (1992).

[16] J. Sapriel, D. Charissoux, V.B. Voloshinov, V.Ya. Molchanov, J. Lightwave Technol. 20, 888 (2002).

[17] V.B. Voloshinov, N.V. Polikarpova, Acustica Acta-Acustica 89, 930 (2003).

[18] V.B. Voloshinov, N.V. Polikarpova, Mol. Quant. Acoust. 24, 225 (2003).

[19] E. Nazarova, A. Tcheryatian, Mol. Quant. Acoust. 28, 259 (2007).

[20] V. Balakshy, A. Vostrikova, Mol. Quant. Acoust. 28, 265 (2007).

[21] V.B. Voloshinov, N.V. Polikarpova, V.G. Mozhaev, Acoustic J. 52, 245 (2006). 